\title{
Student understanding of potential, wavefunctions and the Jacobian in hydrogen in graduate-level quantum mechanics
}

\author{
C. D. Porter, A. Bogdan and A. F. Heckler \\ Dept. of Physics, The Ohio State University, 191 West Woodruff Ave, Columbus, OH 43210
}

\begin{abstract}
This study examined student difficulties related to the potential in the hydrogen atom, and the corresponding ground state, with special attention paid to the role of the Jacobian. The study focused on a population of graduate students at The Ohio State University, and their ability to (1) sketch the approximate potential and radial part of the ground state wavefunction in hydrogen, and (2) their ability to relate this prerequisite knowledge to another relevant quantity: the Bohr radius. Student responses to a sequence of three questions were obtained at the beginning and end of the students' final semester of graduate quantum mechanics. Several prevalent difficulties were identified that persisted to the end of the course, including an inability to sketch the above-mentioned basic features of hydrogen, and a lack of understanding of the Bohr radius.
\end{abstract}

\section{INTRODUCTION}

Quantum mechanics is a notoriously difficult topic in physics [1,2]. Investigation of student difficulties in quantum mechanics has been going on for about two decades [3,4], as has the development of instructional materials to facilitate overcoming those difficulties $[2,4-7]$. Several investigators have studied this at the undergraduate level; less work has been done at the graduate level, although there have been a few pioneering investigations $[2,8,9]$. But the possible misunderstandings constitute a broad space worthy of additional inquiry, and none of the previous studies have addressed student understanding of potentials, wavefunctions, and the Jacobian in the context of hydrogen. The Jacobian is a matrix of partial derivatives relating a transformation of variables. The determinant of this matrix is especially important for integration in different coordinate systems. In the case of hydrogen, the Jacobian determinant for the relevant spherical coordinates is $r^{2} \sin (\theta)$.

The hydrogen atom is an extremely well-known example potential that is suitable both for series solutions and for perturbative treatments. Hydrogen is an integral part of practically all graduate-level quantum mechanics texts (see $[10,11]$ for popular examples). It is therefore reasonable to expect that students with a firm understanding of potential wells and their stationary states should have mastery of this common model system. For this reason the inquiry discussed here is restricted to the hydrogen atom.

Specifically, we seek to answer the following: (1) What basic student difficulties in understanding hydrogen persist into the graduate level? (2) Which difficulties are overcome in the course of graduate studies? (3) Are there aspects of the persisting difficulties that can help us understand why they persist?

\section{METHODS}

As part of an ongoing investigation at The Ohio State University into student difficulties in graduate quantum mechanics, students enrolled in that course were given a 12question written assessment in spring of 2016 (of which only 3 were related to the topic discussed here). The assessment referred to here was newly developed for our study, as the existing metrics did not suit our purposes (see for example $[2,9,12])$. Students were given points in the class equivalent to one homework assignment for participating in the pre- and post-tests at the beginning and end of the semester, each of which was limited to one hour.

The following three questions were repeated verbatim on the pre- and posttests (except for the addition of a figure of the correct wavefunction in the second question in the post-test). Students were given three separate question sheets and turned in answers to one sheet before receiving the next, so that they could not go back to change answers, although they were free to modify part (a) of a question after having worked on part (b).

Question 1: Consider the hydrogen atom. (a) Draw a qualitative plot of the potential which the electron experiences (do not make an effort to include kinetic information, just the electrostatic potential). (b) Now also draw the radial part of the ground state wavefunction (typically denoted $R(r)$ ) of the electron in hydrogen. Again, no numbers are required, just a qualitative sketch showing salient features such as minima, maxima, nodes, asymptotic behavior, etc.

Question 2: While working on homework, your friend correctly states that the radial part of the ground state 
wavefunction of the $\mathrm{H}$ atom is $R(r)=2 a_{0}^{-3 / 2} e^{-r / a_{0}}$. He notes that this is peaked at $r=0$. Then he recalls that the Bohr radius $r=a_{0}$ is the most probable radius of the $\mathrm{H}$ atom. Your friend feels there is a disparity here. Can you help your friend reconcile this information?

Question 3: Consider the spatial wavefunction of a particle in a 3-D spherically symmetric potential well that has not been specified:

$$
\psi(r, \theta, \phi)=\left(\pi \beta^{3}\right)^{-1 / 2} e^{-r / \beta}
$$

Where $\beta$ is some parameter with units of length.

(a) Write an expression for the probability of finding a particle that is in the above state at a distance from the origin in the interval between $r_{0}$ and $r_{0}+d r$.

(b) For what values of $r_{0}$ is the probability from (a) largest?

Question 1 was designed to determine whether the student had a basic conceptual understanding of the radial part of the hydrogen ground state wave function and the corresponding potential. Answers were counted as correct if they showed anything that looked qualitatively like the correct $1 / r$ potential and the $R(r)=\pi^{-1 / 2} a_{0}^{-3 / 2} e^{-r / a_{0}}$ exponential decay of the radial part of the wavefunction. Question 2 was designed to determine whether or not the student could relate this basic knowledge to a relevant length scale (the Bohr radius $a_{0}$ ). In particular, we asked them to resolve an apparent paradox that can arise from incorrect understanding of the basic facts from question 1 . Answers were counted as correct if they made any mention of the Jacobian, "the $r^{2}$ from spherical coordinates" or equivalent. Finally, Question 3 asks students to set up a calculation to determine the probability of finding a particle in some range given a state, and to determine the position of highest probability. Since the given state is the radial part of the ground state of hydrogen (with a change of variable names $a_{0} \rightarrow \beta$ ), this assesses whether students can resolve the problem when it is phrased as a mathematical question, rather than a physical one. The answer to part (a) is $\left(4 / \beta^{3}\right) e^{-2 r / \beta} r^{2} d r$ and the answer to part (b) is $r=\beta$.

\section{RESULTS \& DISCUSSION}

We present our findings from each question separately first, then discuss connections between questions. In all cases, $N=35$ for the pretest and $N=34$ for the posttest (one student dropped; the remaining cohort was unchanged).

Question 1: Most students (54\% pre, 62\% post) correctly answered question 1 (a) (see Figure 1). The most common error was including the centrifugal barrier in the sketch of the potential, despite the question specifying that no kinetic information should be included, and referring to a ground state (which has no angular momentum and therefore no centrifugal barrier). There were a few more serious errors, such as drawings of harmonic oscillator potentials, but these were few. A $\chi^{2}$-test revealed no significant change in correct answers pre-post, with the test statistic: $\chi^{2}(34)=1.4, p=0.25$, and Cohen's $d=0.10$. Nor was there a significant change in the number of students including the centrifugal barrier $\left(\chi^{2}(34)=0.10, p=0.75\right)$.

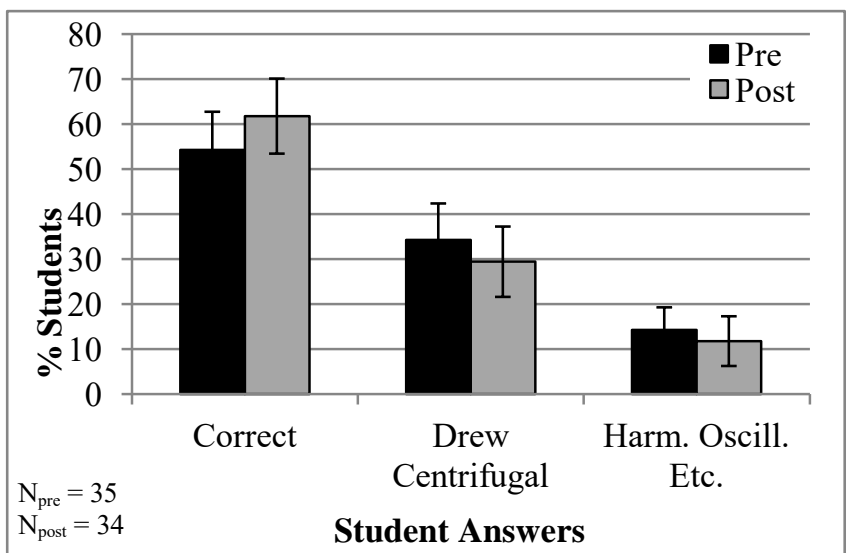

Figure 1: Pre-post comparisons of question 1(a). Changes between pre- and posttest answers were not significant (see text). Error bars indicate standard error. Note that percentages may not add exactly to $100 \%$ in all figures, due to small numbers of students who did not provide any answer, or gave answers that were difficult to categorize. In all cases, the vast majority of student responses are reflected in the figures.

Students performed much more poorly on question 1 (b), with only $17 \%$ and $24 \%$ answering correctly on the pre and posttests respectively (not a significant difference, with $\chi^{2}(34)=2.9, p=0.09$, Cohen's $\left.d=0.10\right)$. Some common errors and their prevalence are shown in Figure 2.

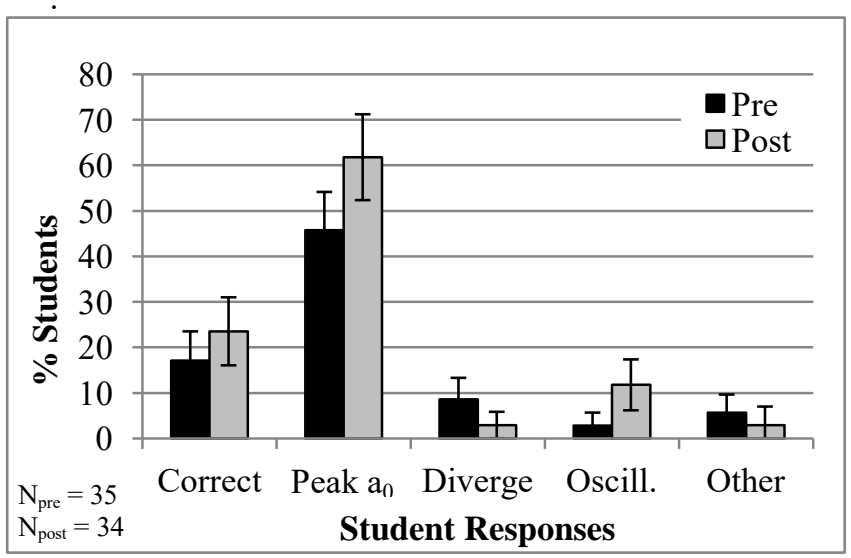

Figure 2: Pre-post comparisons of question 1(b). Error bars indicate standard error. 
By far the most common error was drawing the wavefunction peaked slightly away from zero. Many, but not all students labeled this point $a_{0}$ explicitly, making the incorrect claim that the radial part of the wavefunction is peaked at the Bohr radius. This fraction increased from $46 \%$ to $62 \%$ between pre- and posttests (which is significant, with $\chi^{2}(34)=10, p<0.05$, Cohen's $d=0.33$ ). Again, other errors were present, but in a minority of cases. These included drawing wavefunctions that diverged at the origin, and wavefunctions with nodes.

Question 2: Relatively few students could resolve this apparent paradox $(17 \%$ on the pretest, and $41 \%$ on the posttest, see Figure 3$)$. The increase in correct answers was, however, statistically significant $\left(\chi^{2}(34)=41, p<0.05\right.$, Cohen's $d=0.37)$. Answers were counted as correct if they made any mention of "Jacobian", or "factor of $r^{2}$ ", or equivalent.

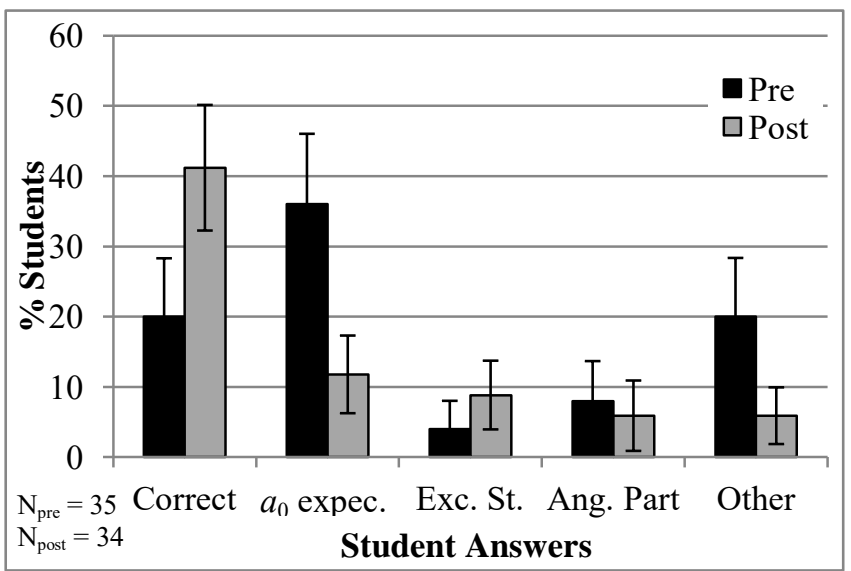

Figure 3: Comparison of certain errors in student responses to question 2. Error bars indicate standard error.

Also significant was the drop in the fraction of students erroneously claiming that the Bohr radius is actually the expectation value of the position. Other common erroneous explanations included: "You are only including the ground state. The inclusion of excited states changes the answer" and "You have only included the radial part of the wavefunction. The angular part of the wavefunction changes the answer". Many students simply wrote that they did not know.

Question 3: More students correctly answered question 3 (a) on the pretest than correctly answered any other question. Also, significantly more answered this correctly on the pretest than on the posttest $\left(\chi^{2}(34)=33, p<0.05\right.$, Cohen's $d=0.40)$. This can be seen in Figure 4.

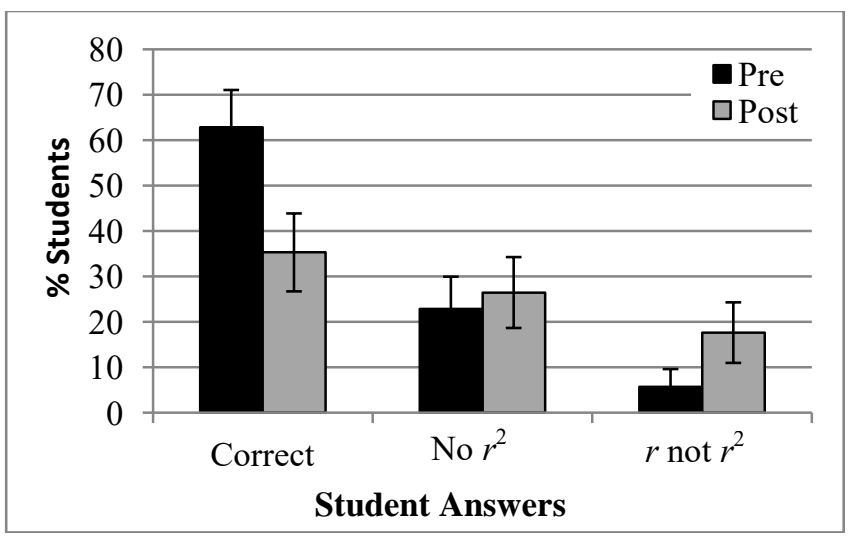

Figure 4: Comparison of 3(a) pre-posttest performance. Error bars indicate standard error.

It is interesting that on the posttest, more students were able to answer question 2 correctly than were able to write out the expression for probability in question 3(a). The most common errors included omitting any factors related to the radius (the Jacobian) or including a factor of $r$ instead of $r^{2}$. In the latter case, some instances seemed to be an error in writing out the Jacobian (for example by collecting the $r$ together with differentials like $r d r \sin (\theta) d \theta d \phi$ ), and in other cases students seemed to be including a factor of $r$ as though calculating an expectation value. This difference in student intent was not always clear.

Student responses to question 3(b) varied widely. A number of students simply stated the correct answer, with a few of those explicitly stating that they see the connection with the hydrogen atom. There was no significant improvement in students' ability to answer this question between pre- and posttests, with the percent answering correctly rising from $34 \%$ to $38 \%\left(\chi^{2}(34)=0.69, p=0.40\right)$. The most common mistake was to make an argument that the most likely position is $r=0$ (29\% pre and $38 \%$ post). This increase was significant $\left(\chi^{2}(34)=4.6, p<0.05\right.$, Cohen's $d=0.13$ ) Some students seemed to arrive at this by a symmetry argument, while others simply took the peak in the wavefunction to be sufficient.

\section{CONCLUSIONS}

The findings above indicate that graduate students have a number of deficits related to hydrogen that may translate to difficulties with other potential wells and their eigenstates. These include fundamental conceptual errors such as diverging (non-normalizable) wavefunctions, as well as a lack of prerequisite knowledge of the hydrogen atom. Posttesting was carried out at the end of students' graduate quantum mechanics instruction. This means that the deficits identified in this work likely persist well into graduate school, if not beyond. Principle observations included: (1) Many students do not know the meaning of 
the Bohr radius, often confusing it with $\langle\hat{r}\rangle$, and sometimes making claims such as "it is the radius of the electron cloud". (2) Many students cannot draw the radial part of the ground state wavefunction of hydrogen, or cannot draw the electrostatic potential in hydrogen. (3) Many students do not know that the Jacobian affects probability distributions, or do not know how it does so.

Some preliminary anecdotal evidence suggests that simply making instructors aware of these subtle misconceptions can go a long way in correcting them, but many of the misunderstandings are difficult to elicit. The confusion about the role of the Jacobian in the hydrogen atom can be especially difficult to unpack. Many students have an incorrect picture of the potential well and ground state. The incorrect picture is self-consistent in that the radial part of the wavefunction is (incorrectly) peaked around $a_{0}$ where they (incorrectly) draw the minimum in the potential that is typically associated with the centrifugal barrier. It is not clear whether, in the majority of cases, the incorrect potential leads students to an incorrect radial part of the wavefunction, or vice versa. Anecdotal evidence suggests that both paths exist, but it is not yet clear which is dominant. The nature of this self-consistency (the specious wavefunction being peaked at $r \neq 0$ ) means that students see no contradiction between the form of the ground state and the value of the most probable proton-electron distance. Even once students are shown the correct wavefunction and potential, they still may not see the role of the Jacobian, because many students say the Bohr radius is the expectation value of $r$, not the most probable value of $r$. Confusion between the closely-related concepts of expectation value and probability density are known at the undergraduate level [13] but not in the context of hydrogen, where the Jacobian can further complicate the picture. Other students, once shown correct potentials and wavefunctions, are unable to explain why the Bohr radius is the most probable radial value when the radial part of the wavefunction is peaked at $r=0$. Discovering these related misunderstandings requires careful inquiry, such as the sequence of questions we asked. One could easily construct a comparable question using a spherical box of finite height.

There is strong indication that even when students answer these questions correctly, their understanding is still flawed. For example, a significantly higher percentage of students correctly answered question 3(a) on the pretest than on the posttest ( $63 \%$ vs. $35 \%)$. On the posttest $41 \%$ of students were able to resolve the paradox in question 2 by invoking the Jacobian, and yet only $35 \%$ of the class could correctly write down a probability involving the Jacobian in question 3(a), indicating their awareness of the Jacobian may be limited to only certain contexts.

The inability of many students to draw the electrostatic potential in hydrogen and/or the radial part of its ground state wavefunction indicates some serious gaps in graduate student understanding of an important quantum system. It is clear that students have a tendency to include the centrifugal barrier in the potential. This is not surprising since many textbooks [10] group the centrifugal term found from the angular part of the wavefunction together with the electrostatic potential to make an "effective potential" for computational convenience. But this is not merely a matter of semantics. We see here that it is not clear to students that this is a part of the kinetic energy associated with angular momentum as opposed to part of the electrostatic potential (both through careful wording of the question and by referring to the ground state, which has no centrifugal barrier).

It is important to note that this investigation was carried on a very small population from a single university. It would be interesting to see if similar difficulties are experienced by students from other institutions, particularly institutions with differently-sized Physics programs.

\section{Acknowledgements}

We acknowledge partial support from the Center for Emergent Materials: an NSF MRSEC under award number DMR-1420451.

\section{References}

[1] D. J. Griffiths, Introduction to Quantum Mechanics $2^{\text {nd }}$ Ed., Prentice Hall, Upper Saddle River, NJ, 1995, Preface.

[2] C. Singh, Am. J. Phys. 76, 277 (2008).

[3] P. Jolly, D. Zollman, S. Rebello, and A. Dimitrova, Am. J. Phys. 66 (1), 57-63 (1998).

[4] L. Bao, P. Jolly, and E. F. Redish, AAPT Announcer 26 (2), 70-71 (July 1996).

[5] M. C. Wittmann, R. N. Steinberg, and E. F. Redish, Am. J. Phys. 70, 218 (2002).

[6] S. Goldhaber, S. Pollock, M. Dubson, P. Beale, and K. Perkins, AIP Conf. Proc. 1179, 145 (2009).

[7] L. Bao and E. F. Redish, Am. J. of Phys. 70, 210 (2002).

[8] C. Singh, Am. J. Phys. 69, 885 (2001).

[9] L.D. Carr and S. B. McKagan, Am. J. Phys. 77, 308 (2009).

[10] R. Shankar, Principles of Quantum Mechanics $2^{\text {nd }} E d$., Springer Science, New York, NY, 1994.

[11] J. J. Sakurai and Jim J. Napolitano, Modern Quantum Mechanics $2^{\text {nd }}$ Ed., Pearson, Essex, England, 2014.

[12] S. Wuttiprom, M. D. Sharma, I. D. Johnston, R. Chitaree and C. Soankwan, Int. J. Sci. Ed., 31:5, 631-654

(2009).

[13] H. Sadaghiani and L. Bao, PER Conf. Proc.818, 61-64

(2005).

[14]G. Passante, P. J. Emigh, and P. S. Shaffer, "Phys. Rev. Sp. Top. - PER 11, 020111 (2014). 